\title{
GAMBLING BEHAVIOR IN ALCOHOL-SERVING AND NON-ALCOHOL-SERVING-VENUES: A STUDY OF ELECTRONIC GAMING MACHINE PLAYER USING ACCOUNT RECORDS
}

Tony Leino ${ }^{1}$, Helge Molde ${ }^{1}$, Mark D. Griffiths ${ }^{2}$, Rune A. Mentzoni ${ }^{3,4}$, Dominic Sagoe ${ }^{3}$, and Ståle Pallesen ${ }^{3}$

1) Department of Clinical Psychology, University of Bergen, Christiesgate 12, N-5015 Bergen, Norway

2) International Gaming Research Unit, Psychology Division, Nottingham Trent University, Burton Street, Nottingham, NG1 4BU

3) Department of Psychosocial Science, University of Bergen, Christiesgate 12, N-5015 Bergen Norway

4) KoRus-Øst, Innlandet Hospital Trust, Norway

Funding: This work was supported by the Research Council of Norway project no. 213757. The funding source had no influence on the study design, data collection and analysis, interpretation of the data, writing of the paper, and the decision to submit the paper for publication.

Acknowledgment: The authors would like to acknowledge Norsk-Tipping, the state owned gambling company in Norway for supplying the data. Norsk-Tipping had no influence on the study design, data collection and analysis, interpretation of the data, writing of the paper, and the decision to submit the paper for publication 


\section{Abstract}

Aim: Contextual factors, such as venue characteristics appear to influence gambling behavior. However, few studies have compared the relationship between gambling behavior in alcohol-serving venues (ASVs) and nonalcohol serving venues (NASVs). The aim of the study was to examine individual gambling behavior in ASVs and NASVs.

Method: A repeated-measures design was used to examine individual gambling behavior in ASVs and NASVs covering a month. The sample comprised 1,452 observations of 726 individuals (25.2\% female). A quantile regression model was conducted to examine individual differences in gambling behavior (number of days, sessions, bets made, stake and time spent, net balance, and average bet size) across ASVs and NASVs. Analyses were broken down by gambler category (those that reached legal mandatory spending limits and those that did not) as well as on time frame (overall gambling behavior and average in-session gambling behavior).

Results: Individuals gambled regularly in NASVs and occasionally in ASVs. Compared to NASVs, in-session gambling behavior was more variable in ASVs. In-session analysis showed that non-limit reaching gamblers staked less money in ASVs than in NASVs but lost more money in ASVs than in NASVs. Limit reaching gamblers showed no differences in gambling behavior across venues.

Conclusion: The findings show that in-session gambling behavior is more variable in ASVs compared to NASVs regardless of gambling category. Non-limit reaching gamblers may be more sensitive to contextual factors than limit reaching gamblers and appear to be more willing to take more risk in ASVs compared to NASVs. The contextual implications are discussed.

Keywords: Contextual characteristics, gambling venues, gambling behavior, account-based gambling data, repeated measures, alcohol. 


\section{Introduction}

Contextual factors, such as venue and structural game characteristics appear to influence gambling behavior (Hing \& Haw, 2010; Marshall, 2009; Parke \& Griffiths, 2007; Young, Markham, \& Doran, 2012). It is well established that density and proximity of gambling venues are positively associated with gambling participation and gambling problems (Abbott, 2007; Ministry of Health, 2008). Moreover, it is assumed that gambling in diffused sites (i.e., venues where gambling is not the main activity within the site) is less intense than gambling within concentrated sites (i.e., venues where gambling is the primary activity of the site) (Reith, 1999).

However, less is known about the relationship between type of venue, venue characteristics, and gambling behavior (Hing \& Haw, 2010; Young et al., 2012). As all gambling venues differ, the individual has the opportunity to choose to gamble in a multitude of different settings, such as casinos, bingo halls, arcades, betting shops, clubs, and pubs. As such, the selection of a specific venue over another might be related to different gambling motives (Thorne, Rockloff, Langham, \& Li, 2016) that may subsequently influence gambling behavior. In line with the proposition that structural game characteristics might influence gambling motives and gambling behaviors (Parke \& Griffiths, 2007), venue characteristics may also have an additional effect on gambling behavior (Griffiths \& Parke, 2003; Markham, Young, \& Doran, 2012). Consequently, the relationship between venue characteristics and gambling behavior merits further research (Hing \& Haw, 2010; Young et al., 2012).

The unique characteristics of a venue have been associated with both choice and popularity of the venue (Hing \& Haw, 2010). The attractiveness of a gambling venue is positively associated with hospitality factors, safety and secure surroundings, atmospheric features (e.g., décor, color, lighting, temperature, floor layout, seating comfort, noise level, ceiling height, etc.), the availability of low denomination electronic gaming machines, low entry costs, and social opportunities (Griffiths, 2009; Hing \& Haw, 2010). The size of a venue appears to influence both those seeking out the venue and the gambling behavior. Large venues (casinos and clubs in shopping centres) have been associated with a higher proportion of problem gamblers (PGs) compared to small venues. Furthermore, individuals are more likely to gamble on EGMs and are more prone to long gambling sessions in casinos compared to other venues (Markham et al., 2012; Young et al., 2012). The relationship between venue size and gambling behavior is possibly due to a greater range of gambling opportunities and a higher proportion of EGMs (Young et al., 2012), but this relationship may also be due to ‘social contagion' where individuals appear to gamble more intensely in the presence of others (Cole et al., 2011; Rockloff \& Dyer, 2007; Rockloff, Greer, \& Fay, 2011). In contrast, it has also been reported that non-casino 
Hodgins, 2012). The authors hypothesized that this may be due to the fact that smaller venues are typically more widespread and therefore more easily accessible compared to casinos. Furthermore, there might be motivational differences to visit larger gambling venues compared to smaller venues. The prime motive to visit a large venue might be gambling, whereas visiting a pub or club with gambling opportunities might primarily be related to other factors, such as to be socially active (Clarke et al., 2012; Reith, 1999; Sévigny et al., 2016). For example, a recent Canadian study reported that in small gambling venues (5 to 10 EGMs), players had a longer gambling history, gambled more sessions per month, had higher stakes per gambling session, were more motivated to gamble for money, and were four times more likely to a problem gambler compared to those playing in large gambling venues (335 EGMs) (Sévigny et al., 2016). In contrast, large gambling venue players spent more time per gambling session, preferred to gamble undisturbed, and were more motivated to gamble for pleasure. Small venue players were more likely to emphasise the venue as a social arena and were more likely to both drink alcohol and consume greater amounts of alcohol while they gambled compared to large venue players.

In the psychological literature on the co-occurrence of gambling and drinking alcohol, it has been suggested that all types of gamblers may become more motivated to gamble following the ingestion of alcohol (Dickerson \& O'Connor, 2011). Indeed, intoxication appears to increase the motivation to gamble (Barrett, Collins, \& Stewart, 2015). The effect of alcohol on gambling behavior has received much attention in the gambling literature although almost all of these studies are single cross-sectional designs. Bearing in mind this major methodological limitation, research has demonstrated that alcohol consumption is related to more persistent gambling behavior among pathological gamblers (e.g., Ellery, Stewart, \& Loba, 2005), regular gamblers (e.g., Kyngdon \& Dickerson, 1999), and young adults (e.g., Cronce \& Corbin, 2010). However, surveys (e.g., Markham et al., 2012) and observational studies (e.g., Baron \& Dickerson, 1999) have also reported that the number of drinks consumed is negatively associated with the duration of a gambling session and decreases the probability of EGM gambling among non-problem gamblers (Markham et al., 2012). Furthermore, although regular alcohol consumption increases the likelihood to gamble, the likelihood of consuming alcohol has been shown not to increase with gambling (Bussu \& Detotto, 2015). As such, avoiding drinking alcohol while gambling may be an indication of a responsible gambler, whereas drinking while gambling may be an indication of a 'reckless' gambler (Welte, Barnes, Wieczorek, \& Tidwell, 2004). In sum, gambling behavior appears to differ between venues, which appears be related to different gambling motives and different gambling contexts. 
Previous studies on how venue characteristics influence gambling behavior have all been based on selfreport-methods (e.g., surveys), field observations, and laboratory experiments - all of which are associated with different limitations. Observational studies are often based on small samples over a relatively short time span. In self-report studies, the relationship between subjective experiences and objective recordings are often weak, (Schwarz \& Strack, 1999) which may be explained by mood and recall biases associated with the former as well as biases related to self-representation (Schwarz \& Strack, 1999). Laboratory studies have been criticized for having low external validity due to 'hidden’ contextual factors and an artificial environment (Anderson \& Brown, 1984).

Another approach than those referred to above would be to examine actual individual gambling behavior directly in different contexts using account-based (i.e., behavioral tracking) player data. Account-based player gambling entails registering of gambling behavior that is linked to an individual account (Gainsbury, 2011). The method provides a totally objective account of individual gambling behavior collected electronically and unobtrusively in the natural gambling environment of the individual (Griffiths, 2014). As such, the method offers a unique possibility to examine individual gambling behavior in different contexts. In fact, recent research using behavioral tracking technologies has demonstrated that the more that a person gambles, the greater disparity between their self-reported behavior and their actual behavior (Braverman et al., 2014; Auer \& Griffiths, 2016a).

An understanding of gambling behavior in different settings would add further understanding of how contextual factors influence gambling behavior and can also help inform the extent to which different settings are associated with more risky gambling behavior compared to other settings. Alcohol serving venues (ASVs) and non-alcohol-serving venues (NASVs) represent two different gambling contexts that have not previously been compared using account-based player data. Thus, the purpose of the present study was to explore individual gambling behavior in alcohol serving venues (ASVs) and non-alcohol-serving venues (NASVs) using a repeated samples design based on account-based gambling data.

\section{Method}

The study in context: Behavioral tracking data from Norwegian Multix gamblers were obtained from Norsk-Tipping, the national gambling company in Norway. Multix is a fully digitalized multigame networkbased video lottery terminal (VLT). All terminals are similar and offer the same games. An individual player card linked to a personal player account is required to gamble on Multix. Since all Multix terminals are interconnected, individual gambling behavior and gambling responses on any gambling terminal can be tracked over time. In an attempt to limit excessive gambling behavior, Multix incorporates mandatory time and loss 
GAMBLING BEHAVIOR IN ALCOHOL AND NON-ALCOHOL VENUES 6

limits set by Norwegian legislative authorities. The maximum loss limit was 600 NOK per day and/or 2,500

NOK per month (1USD $\approx 6$ NOK) in 2013. In addition, a 10-minute 'cool-off period' is required after 60 minutes of continuous gambling. If a gambler exceeds these limits, further gambling is not possible on any Multix terminal in that day, that month, or for a period of ten minutes, depending on which limit has been exceeded (Ministry of Culture and Church Affairs, 2008). Although naturally occurring gambling behavior, particularly among problem gamblers, is restricted by these structural limitations, they most likely do not influence or distort individual gambling behavior among the majority of gamblers who gamble in ASVs and NASVs. It should also be noted that land-based gambling is strictly regulated in Norway, and there are no casinos. Multix is located at service stations ( $\mathrm{N}=926$, including convenience stores, kiosks and petrol stations), outdoor and social locations ( $\mathrm{N}=45$, including pubs, bars, hotels, bowling halls - all having alcohol serving permit), grocery stores $(\mathrm{N}=7)$ and locations not included in the first three categories $(\mathrm{N}=11$, including a bookstore, horseracing tracks and other locations). Outside of bingo halls, they are the only permitted VLTs in Norway. Multix terminals located at outdoor and social venues are the only ones with permission to sell alcohol (Norsk-Tipping, personal communication, January 13, 2017).

Design and sample: A repeated-measures design was used to examine individual gambling behavior in ASVs and NASVs over a one-month period. The data reflect natural individual gambling behavior collected unobtrusively across the venues. The sample comprised all individuals who had gambled at least once in both types of venues in a month (May, 2015). Overall, 1,452 observations of 726 individuals (25.2\% female) were analyzed. The mean age of the gamblers was 50.3 years ( $S D=14.9)$ and ranged between 18 and 92 years. In May 2015 there were 48,222 unique active Multix players where 726 Multix players gambled in both ASVs and NASVs. A total of 45 of 989 gambling locations had permission to sell alcohol throughout Norway (NorskTipping, personal communication, 10.01.2017).

Gambling measures: For each venue, individual gambling measures comprised number of days, number of sessions, number of bets made, total stake (in NOK), amount of time spent (in seconds), net balance, and average bet size. Net balance was the difference between the total amount won or lost and total stake. Mean bet size (bet size) was calculated as total stake divided by number of bets made in a session.

Statistical procedure: For ASVs and NASVs, respectively, gambling behavior was analyzed as: (i) overall individual gambling behavior during the one-month period, and (ii) mean gambling behavior per session. Mean gambling behavior per session was calculated as monthly gambling behavior divided by the number of gambling sessions. Analyses were divided between individuals who reached the loss limit (limit reaching gamblers) and 
those who did not (non-limit reaching gamblers). Due to a high variability and non-normality in the data, a quantile regression model (QRM) with robust standard errors was utilized to examine the median difference between individual gambling behavior in ASVs and NASVs. Effect sizes ( $r$ ) were computed by dividing the absolute z value by the square root of $N$ and interpreted using Cohen's criteria (1988) where $.1=$ small effect, .3 $=$ medium effect, and .5 = large effect (Pallant, 2007). The data were analyzed using Stata, version 13 (StataCorp, 2013).

Ethical considerations: Norsk-Tipping, the state owned gambling company in Norway provided the data. The consent to use the data anonymously is covered in contracts between individual gamblers and NorskTipping. To ensure anonymity, Norsk-Tipping created new identity codes for each participant and the key to reversing the process was deleted prior to data export. Furthermore, Norsk-Tipping did not have any input in the research process or writing and stated no constraints on publishing and did not provide any form of funding for the project. The board for Research and Research training at the University of Bergen also reviewed and granted their approval of the project.

\section{Results}

A total of 88 gamblers (12.1\%) reached the monthly loss-limit on Multix VLTs. Neither gender $\left[\chi^{2}(1)=\right.$ $.69, p<.41]$, nor age $[\mathrm{t}(726)=1.33, p<.19$ (two-tailed) $]$ were associated with reaching the monthly loss-limit. Consequently, no separate sub-analyses for gender and age were conducted.

Table 1 shows the mean gambling behavior in May 2015 in ASVs and NASVs independent of individuals. The table shows that more Multix terminals were located and more individuals gambled in NAVs compared to ASVs. Furthermore, gambling participation was more intense in NASVs compared to ASVs on all gambling indices.

Table 2 shows the mean gambling behavior in a one-month period in ASVs and NASVs as well as the results from the QRM analysis. Table 2 also shows the relative frequency of gambling behavior at NASVs and ASVs over a month. The QRM analysis demonstrates that all individuals gambled more days, more sessions, made more bets, and had higher stakes and spent more time in NASVs compared to ASVs. Additionally, all individuals lost less in ASVs. Weak to strong effect sizes in terms of differences in gambling behavior across venues were observed. The effect sizes appeared to be roughly similar among non-limit reaching gamblers and limit reaching gamblers, except for money lost, where the effect of net-loss was less apparent among non-limit reaching gamblers compared to limit reaching gamblers. Hence, on average all individuals lost more money in 
NASVs. Furthermore, loss-limited gamblers lost more money than non-loss-limited gamblers and even more so in NASVs.

Table 3 shows the average gambling behavior per session in ASVs and NASVs and the results from the QRM analysis. The standard deviation was larger in ASVs compared to NASVs across all gamblers, indicating that average gambling behavior is more diverse in ASVs. The QRM analysis showed more bets, higher stakes, and more time spent gambling in NASV among non-limit reaching gamblers. However, non-limit reaching gamblers lost more money in ASVs than in NASVs, whereas average bet size did not differ between venues. Among limit reaching gamblers, the QRM analysis showed no difference in number of bets made, amount of time spent gambling, amount of money staked, and losses between the two types of venues. Only weak differences were observed in gambling behavior between ASVS and NASVs.

\section{INSERT TABLES 1 AND 2 ABOUT HERE}

\section{Discussion}

The present study examined the contextual effect of gambling behavior in ASVs and NASVs by using a quantile regression model based on account-based individual gambling data. Overall, gamblers played more frequently and spent more money in NASVs than in ASVs over the month. This probably reflects the fact that the majority of individuals visit ASVs less frequently and maybe only on special occasions. However, average in-session gambling behavior showed more variation in ASVs among all gamblers. In addition, non-limit reaching gamblers made fewer bets, staked less money, and spent less time gambling, but lost more on average in ASVs within a session. No such differences were observed among limit reaching gamblers between venue types. As such, the results may indicate that non-limit reaching gamblers are more sensitive to contextual variations compared to limit reaching gamblers.

Although average bet size did not vary across venues, non-limit reaching gamblers placed more bets, staked more money, and spent more time in a session in NASVs but lost more in ASVs. Overall, these findings are consistent with a contextual perspective showing that non-limit reaching gamblers are sensitive to contextual factors. The results also show that average in-session gambling behavior in general was more variable in ASVs compared to NASVs. Although speculative, as gambling behavior is more homogenous in NASVs, combined with a generally higher gambling participation found in NASV, this might suggest that individuals are primarily more motivated to gamble on Multix VLTs when visiting a NASV compared to ASV. This is in line with the 
notion that gambling in diffused sites (where gambling is not the primary activity of the venue) are less intense than gambling within concentrated sites where gambling is the primary activity of the venue (Reith, 1999).

As such, the prime motivation why an individual visit an ASV might not be related to Multix gambling only, but also to other reasons (i.e., social). Counter-intuitively, individuals lost more money in ASVs. Higher losses might be an indication of higher risk-taking, which may be related to overall alcohol consumption. This concurs with Cronce and Corbin (2010) and Kyngdon and Dickerson (1999) who found that gamblers lost their money more rapidly when under the influence of alcohol. This could relate to the average bet size because findings suggest there are larger average bet sizes for gamblers under the influence of alcohol than compared to gamblers in a sober state (Cronce \& Corbin, 2010). However, in contrast to Cronce and Corbin (2010), the average bet size did not differ between types of venue in the present study. However, this might be related to the operationalization of bet size. Because Multix offers the gambler several different types of game, the current conceptualization of bet size reflects the overall bet size across all games played. As such, the conceptualization of bet size in the present study might therefore be sub-optimal given that an individual might gamble on different games in alcohol-serving venues and non-alcohol-serving-venues. Another explanation might be related to 'social contagion' and the presence of friends as it has been shown that the presence of other can lead to more intense gambling behavior (Cole et al., 2011; Rockloff \& Dyer, 2007; Rockloff et al., 2011).

In contrast, limit reaching gamblers did not differ on any gambling behaviors or money lost across venues. This is inconsistent with Ellery et al. (2005) who found that probable pathological gamblers spent more time gambling when alcohol intoxicated, but consistent with Markham et al. (2012) who reported that gambling behavior among problem gamblers did not differ by different contexts. However, as gambling behavior was more variable in ASVs compared to NASVs, this may indicate that limit reaching gamblers also are affected by contextual factors, but less so than non-limit reaching gamblers. As such, the findings appear to be partly consistent with a situational normative perspective (see above). It is reasonable to assume that loss-limited gambling is a proxy of gambling problems, hence the results suggest that individuals experiencing gambling problems are less sensitive to context and are more strongly motivated by the specific gambling activity.

Although significant differences in NASVs and ASVs were obtained, the effect sizes were low. This suggests that the contextual differences are marginal among non-limit-reaching gamblers. However, gamblers comprise a heterogeneous group of individuals (Dickerson, Hinchy, England, Fabre \& Cunningham, 1992; Dickerson, 1993) whereas the present study treated gamblers homogenously, making no distinctions except between limit-reaching and non-limit-gamblers. Furthermore, Gainsbury and Blaszczynski (2011) compared a student sample in a 
laboratory setting to club patrons in a gambling venue. Although, the direction of the findings was similar, the field study showed fewer significant results. In summary, the low effect sizes found between the venue type and gambling behavior might be a result of unaccounted individual and/or contextual effects.

\section{Psychosocial implications and future studies}

The findings of the present study may have implications for social policy and development of effective harmminimization strategies. The findings show that non-limit reaching gamblers appear to be more sensitive to contextual factors compared to limit reaching gamblers. Although speculative, this suggests that the availability of alcohol in a gambling venue influences gambling behavior and potentially leads to more risky behavior as indicated by the higher losses in ASVs compared to NASVs among non-limit reaching gamblers. For some individuals, higher losses might serve as a pathway to future gambling problems as they might chase their losses (Lesieur, 1977). As such, the observation that gambling behavior is more variable and associated with higher losses where alcohol is available should be taken into consideration by regulators.

A potentially harm-reducing strategy could be to increase awareness of the problems associated with cooccurring gambling and alcohol use by introducing warnings to the gamblers in ASVs. In addition, because behavioral tracking can be used to monitor individual gambling expenditure and losses, gamblers could be personally notified about their different gambling behavior across contexts. Recent evidence suggests that providing personalized feedback to gamblers helps them reduce the time and money spent gambling (Auer \& Griffiths, 2015; 2016b). Furthermore, individuals should be given the opportunity to set context specific losslimits. In addition, since all Norwegian EGMs are interconnected, one potential solution could be to have different loss-limits based on gambling venue characteristics. For example, the daily mandatory loss limit in ASVs could be set lower than that of NASVs. Based on the findings, a lower mandatory loss-limit might have a beneficial impact on non-loss reaching gamblers as it would limit their losses. Future research could pursue such initiatives empirically in both laboratory and real world settings, examining both their individual and combined effect on gambling behavior.

\section{Limitations and strengths}

Some limitations should be considered when interpreting the results of the present study. Because no measure of alcohol consumption was available, the results should not be interpreted as an indication of the effect of intoxication, but as an indication of the influence of contextual variables on gambling behavior. For example, the differences and variability found in ASV might be due to contextual, alcohol, or social factors, or the 
GAMBLING BEHAVIOR IN ALCOHOL AND NON-ALCOHOL VENUES 11

combined effect of these. As such, it should be noted that there may be other differences between venue types than just the presence or absence of alcohol that could impact on gambling behavior. Furthermore, the results should be viewed as an indication of gambling behavior among gamblers who gamble in both NASVs and ASVs and not as general gambling behavior. It would also have been useful to have a more precise breakdown of the specific venues but these data were not provided by the gaming operator. Despite these limitations, the present study has a number of strengths that deserve mention. To our knowledge, and in contrast with previous studies examining the contextual effects of gambling behavior, this is the first ever study that uses a repeated-measures design and actual gambling data collected unobtrusively comparing gambling behavior among the population of gamblers who have played across different gambling contexts. As such, the study has high ecological and population validity and overcomes many of the weaknesses found in other methods (i.e., laboratory experiments, self-report studies, and observational studies).

\section{Conclusion}

Contextual factors have long been shown to influence gambling behavior (Griffiths \& Parke, 2003). By using account-based individual gambling data and a repeated-measures design, the present study demonstrates that gambling behavior is more variable in ASVs than in NASVs. In contrast to limit reaching gamblers where no contextual effects were observed, non-limit reaching gamblers spent less money, but lost more money in ASVs compared to NASVs. Consequently, non-limit reaching gamblers may be more sensitive to contextual factors compared to limit reaching gamblers. 
GAMBLING BEHAVIOR IN ALCOHOL AND NON-ALCOHOL VENUES 12

\section{References}

Abbott, M. W. (2007). Situational factors that affect gambling behavior. In G. Smith, D. Hodgins, \& R. Williams (Eds.), Research and measurment issues in gambling studies (pp. 251-278). Amsterdam: Elsevier Academic Press.

Anderson, G., \& Brown, R. (1984). Real and laboratory gambling, sensation-seeking and arousal. British Journal of Psychology, 75(3), 401-410. doi/10.1111/j.2044-8295.1984.tb01910.x/pdf

Auer, M., \& Griffiths, M. D. (2015). The use of personalized behavioral feedback for online gamblers: an empirical study. Frontiers in Psychology, 6, 1406. doi:10.3389/fpsyg.2015.01406

Auer, M. \& Griffiths, M. D. (2016a). Self-reported losses versus actual losses in online gambling: An empirical study. Journal of Gambling Studies. Epub ahead of print. doi: 10.1007/s10899-016-9648-0

Auer, M. \& Griffiths, M. D. (2016b). Personalized behavioral feedback for online gamblers: A real world empirical study. Frontiers in Psychology, 7, 1875. doi: 10.3389/fpsyg.2016.01875

Baron, E., \& Dickerson, M. (1999). Alcohol consumption and self-control of gambling behaviour. Journal of Gambling Studies, 15(1), 3-15. doi:10.1023/A:1023057027992

Barrett, S. P., Collins, P., \& Stewart, S. H. (2015). The acute effects of tobacco smoking and alcohol consumption on video-lottery terminal gambling. Pharmacology Biochemistry and Behavior, 130, 3439. doi:10.1016/j.pbb.2014.12.015

Braverman, J., Tom, M. A., \& Shaffer, H. J. (2014). Accuracy of self-reported versus actual online-gambling wins and losses. Psychological Assessment, 26, 865-877. doi: 10.1037/a0036428

Bussu, A., \& Detotto, C. (2015). The bidirectional relationship between gambling and addictive substances. International Gambling Studies, 15(2), 285-308. doi:10.1080/14459795.2015.1042493

Clarke, D., Pulford, J., Bellringer, M., Abbott, M., \& Hodgins, D. C. (2012). An exploratory study of problem gambling on casino versus non-casino electronic gaming machines. International Journal of Mental Health and Addiction, 10(1), 107-121. doi:10.1007/s11469-010-9306-1

Cohen, J. (1988). Statistical power analysis for the behavioral sciences ( $2^{\text {nd }}$ ed.). Hillsdale, NJ: Lawrence Erlbaum Associates.

Cole, T., Barrett, D. K. R., Griffiths, M. D. (2011). Social facilitation in online and offline gambling: A pilot study. International Journal of Mental Health and Addiction, 9, 240-247. doi: 10.1007/s11469-0109281-6 
Cronce, J. M., \& Corbin, W. R. (2010). Effects of alcohol and initial gambling outcomes on within-session gambling behavior. Experimental and Clinical Psychopharmacology, 18(2), 145-157. doi:10.1037/a0019114

Dickerson, M., Hinchy, J., England, S. L., Fabre, J., \& Cunningham, R. (1992). On the determinants of persistent gambling behaviour. I. High-frequency poker machine players. British Journal of Psychology, 83(2), 237-248. doi:10.1111/j.2044-8295.1992.tb02438.x

Dickerson, M. (1993). Internal and external determinants of persistent gambling: Problems in generalising from one form of gambling to another. Journal of Gambling Studies 9(3), 225-245. doi:10.1007/BF01015920

Dickerson, M., \& O'Connor, J. (2011). Gambling as an addictive behaviour: Impaired control, harm minimisation, treatment and prevention: Cambridge University Press.

Ellery, M., Stewart, S. H., \& Loba, P. (2005). Alcohol's effects on video lottery terminal (VLT) play among probable pathological and non-pathological gamblers. Journal of Gambling Studies, 21(3), 299-324. doi:10.1007/s10899-005-3101-0

Gainsbury, S. (2011). Player account-based gambling: potentials for behaviour-based research methodologies. International Gambling Studies, 11(2), 153-171. doi:10.1080/14459795.2011.571217

Gainsbury, S., \& Blaszczynski, A. (2011). The appropriateness of using laboratories and student participants in gambling research. Journal of Gambling Studies, 27(1). 83-97. doi:10.1007/s10899-010-9190-4

Griffiths, M. D. (2009). Casino design: Understanding gaming floor influences on player behaviour. Casino and Gaming International, 5(1), 21-26.

Griffiths, M. D. (2014). The use of behavioural tracking methodologies in the study of online gambling. SAGE research methods cases. London: Sage.

Griffiths, M. D. \& Parke, J. (2003). The environmental psychology of gambling. In G. Reith (Ed.), Gambling: Who wins? Who loses? (pp. 277-292). New York: Prometheus Books.

Hing, N., \& Haw, J. (2010). The influence of venue characteristics on a player's decision to attend a gambling venue: Final report, for Gambling Research Australia, Centre for Gambling Education \& Research, Southern Cross University, Lismore, NSW.

Kyngdon, A., \& Dickerson, M. (1999). An experimental study of the effect of prior alcohol consumption on a simulated gambling activity. Addiction, 94(5), 697-707. doi:10.1046/j.1360-0443.1999.9456977.x

Lesieur, H. R. (1977). The chase: Career of the compulsive gambler. Garden City, NY: Anchor Press. 
Markham, F., Young, M., \& Doran, B. (2012). The relationship between alcohol consumption, gambling behaviour and problem gambling during a single visit to a gambling venue. Drug and Alcohol Review, 31(6), 770-777. doi:10.1111/j.1465-3362.2012.00430.x

Marshall, D. (2009). Gambling as a public health issue: The critical role of the local environment. Journal of Gambling Issues, 23, 66-80. doi/pdf/10.4309/jgi.2009.23.4

Ministry of Culture and Church Affairs. (2008). Spilleregler for Multix.

Ministry of Health. (2008). Raising the odds? Gambling behaviour and neighbourhood access to gambling venues in New Zealand. Wellington, NZ: Ministry of Health.

Pallant, J. (2007). SPSS survival manual: A step by step guide to data analysis using SPSS for Windows (3 ed.). Maidenhead: Open University Press.

Parke, J., \& Griffiths, M. D. (2007). The role of structural characteristics in gambling. In G. Smith, D. Hodgins, \& R. Williams (Eds.), Research and measurement issues in gambling studies (pp. 218-250). Amsterdam: Elsevier/Academic Press.

Reith, G. (1999). The age of chance: Gambling in Western culture. London: Routledge.

Rockloff, M. J., \& Dyer, V. (2007). An experiment on the social facilitation of gambling behavior. Journal of Gambling Studies, 23(1), 1-12. doi:10.1007/s10899-006-9042-4

Rockloff, M. J., Greer, N., \& Fay, C. (2011). The social contagion of gambling: How venue size contributes to player losses. Journal of Gambling Studies, 27(3), 487-497. doi:10.1007/s10899-010-9220-2

Schwarz, N., \& Strack, F. (1999). Reports of subjective well-being: Judgmental processes and their methodological implications. In D. Kahnemann, E. Diener, \& N. Schwarz (Eds.), Well-being: The foundations of hedonic psychology (pp. 61-84). New York: Russel Sage Foundation.

Sévigny, S., Leclerc, M., Goulet, A., Côté, K., Jacques, C., Ladouceur, R., \& Giroux, I. (2016). Electronic gambling machine gamblers' characteristics vary according to the type of gambling venue: A Canadian study. International Gambling Studies, 16(1), 116-139. doi:10.1080/14459795.2016.1151912

StataCorp. (2013). Stata statistical software: Release 13. College Station, TX: StataCorp LP.

Thorne, H. B., Rockloff, M. J., Langham, E., \& Li, E. (2016). Hierarchy of gambling choices: A framework for examining EGM gambling environment preferences. Journal of Gambling Studies, 32(4), 1101-1113. doi:10.1007/s10899-016-9601-2 
GAMBLING BEHAVIOR IN ALCOHOL AND NON-ALCOHOL VENUES 15

Welte, J. W., Barnes, G. M., Wieczorek, W. F., \& Tidwell, M. C. (2004). Simultaneous drinking and gambling: a risk factor for pathological gambling. Substance Use \& Misuse, 39(9), 1405-1422. doi.org/10.1081/JA120039397

Young, M., Markham, F., \& Doran, B. (2012). Placing bets: Gambling venues and the distribution of harm. Australian Geographer, 43(4), 425-444. doi:10.1080/00049182.2012.731302 
Table 1. Descriptive statistics of overall gambling behaviour in non-alchohol serving and alcohol serving venues.

\begin{tabular}{|c|c|c|c|c|c|}
\hline \multirow{3}{*}{ \# Terminal } & & Mean & SD & Md & Range \\
\hline & Non-alchohol serving venue & 2.9 & 1.6 & 2 & $1-12$ \\
\hline & Alcohol serving venue & 1.8 & .98 & 2 & $1-6$ \\
\hline \multirow{2}{*}{ \# Gamblers } & Non-alchohol serving venue & 119.4 & 92.0 & 99 & $2-688$ \\
\hline & Alcohol serving venue & 24.2 & 29.8 & 16 & $2-147$ \\
\hline \multirow{2}{*}{ Days } & Non-alchohol serving venue & 362.5 & 279.2 & 302 & $3-1,785$ \\
\hline & Alcohol serving venue & 65.1 & 89.5 & 40 & $3-454$ \\
\hline \multirow{2}{*}{ Sessions } & Non-alchohol serving venue & $1,516.9$ & $1,401.0$ & 1,112 & $5-10,766$ \\
\hline & Alcohol serving venue & 301.8 & 464.7 & 115 & $3-2028$ \\
\hline \multirow{2}{*}{ Bets made } & Non-alchohol serving venue & $87,157.2$ & $80,146.1$ & 63,432 & $16-571,649$ \\
\hline & Alcohol serving venue & $16,885.9$ & $27,876.5$ & 6,492 & $118-144,058$ \\
\hline \multirow{2}{*}{ Stake } & Non-alchohol serving venue & $608,728.7$ & $546,866.9$ & 452,754 & 72 - 3,818,297 \\
\hline & Alcohol serving venue & $123,915.7$ & $242,992.6$ & 37,681 & $1290-1,429,544$ \\
\hline \multirow{2}{*}{ Time spent } & Non-alchohol serving venue & $502,562.4$ & $459,027.3$ & 367,991 & $386-3,407,675$ \\
\hline & Alcohol serving venue & $102,929.0$ & $173,863.6$ & 37,887 & 1405 - 928,202 \\
\hline \multirow{2}{*}{ Money lost } & Non-alchohol serving venue & $-48,362.7$ & $44,228.9$ & $-35,652.3$ & $-290,548.5-7081.5$ \\
\hline & Alcohol serving venue & $-10,002.15$ & $19,246.07$ & $-4,570.95$ & $-108,380.6-4,965.5$ \\
\hline
\end{tabular}

Commented [SP1]: Perhaps write «Md = median» below this and other relevant tables? 
GAMBLING BEHAVIOR IN ALCOHOL AND NON-ALCOHOL VENUES 17

Table 2. A quantile regression model comparing overall gambling behavior in NASV and ASV.

\begin{tabular}{|c|c|c|c|c|c|c|c|c|c|c|c|c|c|}
\hline & & \multicolumn{4}{|c|}{ Non-alchohol serving venues } & \multicolumn{4}{|c|}{ Alchohol serving venues } & \multirow[b]{2}{*}{$\Delta$} & \multirow[b]{2}{*}{$\mathrm{Z}$} & \multirow[b]{2}{*}{$95 \% \mathrm{CI}$} & \multirow[b]{2}{*}{$\mathrm{r}$} \\
\hline \multirow{3}{*}{ Days } & & Mean & SD & Md & MAD & Mean & SD & Md & MAD & & & & \\
\hline & Non-limit reaching gamblers & 7.2 & 6.1 & 5 & 3 & 2.7 & 3.4 & 1 & 0 & -4 & -16.19 & {$[-4.48,-3.52]$} & 0.64 \\
\hline & Limit reaching gamblers & 7.8 & 4.6 & 7 & 3 & 3.2 & 2.8 & 2 & 1 & -5 & -6.51 & {$[-6.51,-3.49]$} & 0.69 \\
\hline \multirow{2}{*}{ Sessions } & Non-limit reaching gamblers & 39.1 & 80.1 & 16 & 13 & 13.9 & 36.5 & 4 & 3 & -12 & -9.53 & {$[-14.47,-9.53]$} & 0.38 \\
\hline & Limit reaching gamblers & 38.1 & 52.3 & 20 & 13 & 13.2 & 16.8 & 7 & 6 & -13 & -4.11 & {$[-19.20,-6.80]$} & 0.44 \\
\hline \multirow{2}{*}{ Bets made } & Non-limit reaching gamblers & $1,993.5$ & $2,637.0$ & 1020 & 846 & 750.4 & $1,491.8$ & 235 & 205 & -785 & -9.62 & {$[-945,05,-624.95]$} & 0.38 \\
\hline & Limit reaching gamblers & $2,380.0$ & 3,358.5 & 1119 & 685 & 787.2 & 938.5 & 363 & 289 & -756 & -2.89 & {$[-1,268.32,-243.68]$} & 0.31 \\
\hline \multirow{2}{*}{ Stake } & Non-limit reaching gamblers & $12,626.5$ & $17,069.7$ & 7,005 & 5,391 & 5,137 & 10,511 & 1,514 & 1,321 & $-5,490$ & -11.77 & {$[-6,404.79,-4,576.81]$} & 0.47 \\
\hline & Limit reaching gamblers & $13,802.7$ & $12,088.9$ & 10,110 & 5,699 & 5,959 & 7,198 & 3,080 & 2,021 & $-7,030$ & -4.91 & {$[-9,835.64,-4,224.36]$} & 0.52 \\
\hline \multirow{2}{*}{ Time spent } & Non-limit reaching gamblers & $11,487.4$ & $15,144.6$ & 5,951 & 4,882 & 4,534 & 8,970 & 1,389 & 1,227 & $-4,562$ & -9.14 & {$[-5,540.14,-3,583.86]$} & 0.36 \\
\hline & Limit reaching gamblers & $13,385.4$ & $17,823.6$ & 5,971 & 4,069 & 4,610 & 5,770 & 2,163 & 1,697 & $-3,808$ & -2.60 & {$[-6,681.32,-934.68]$} & 0.28 \\
\hline \multirow{2}{*}{ Net-balance } & Non-limit reaching gamblers & 590.5 & $1,582.6$ & -606 & 697 & -322.4 & 1,080 & -247 & 281 & 359 & 5.18 & {$[223.20,495.00]$} & 0.21 \\
\hline & Limit reaching gamblers & $1,881.3$ & $1,116.8$ & $-1,947$ & 526 & -618.8 & 1,117 & -554 & 496 & 1,393 & 7.20 & {$[1,013.78,1,772.22]$} & 0.77 \\
\hline
\end{tabular}

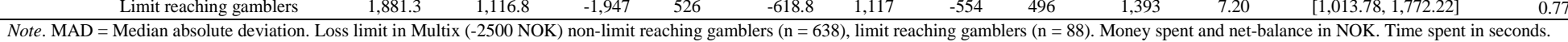

Average bet size $=$ Money spent $/$ bets made. All $p<.001$. $\Delta=$ Difference between MD in Non-alcohol serving venues and MD in Alcohol serving venues and subsequent significance test and $95 \% \mathrm{CI}$ of this difference. 
Table 3. A quantile regression model comparing gambling behavior in NASV and ASV within a session.

\begin{tabular}{|c|c|c|c|c|c|c|c|c|c|c|c|c|c|}
\hline & & \multicolumn{4}{|c|}{ Non-alchohol serving venues } & \multicolumn{4}{|c|}{ Alchohol serving venues } & \multirow[b]{2}{*}{$\Delta$} & \multirow[b]{2}{*}{$\mathrm{Z}$} & \multirow[b]{2}{*}{$95 \% \mathrm{CI}$} & \multirow[b]{2}{*}{$\mathrm{r}$} \\
\hline \multirow{3}{*}{ Bets made } & & Mean & SD & Md & MAD & Mean & SD & $\mathrm{Md}$ & MAD & & & & \\
\hline & Non-limit reaching gamblers & 64.9 & 46.2 & 54 & 23 & 66.4 & 68.9 & 47 & 25 & -7.3 & -3.00 & {$[-12.09,-2.53]$} & 0.12 \\
\hline & Limit reaching gamblers & 72.2 & 43.6 & 60 & 18 & 71.0 & 53.2 & 54 & 20 & -5.7 & 1.07 & {$[-16.22,4.79]$} & 0.11 \\
\hline \multirow{2}{*}{ Stake } & Non-limit reaching gamblers & 494.4 & 483.2 & 362 & 193 & 510.6 & 672.1 & 300 & 183 & -60.0 & 2.54 & {$[-106,-13.70]$} & 0.10 \\
\hline & Limit reaching gamblers & 632.3 & 507.9 & 490 & 252 & 622.7 & 562.2 & 440 & 267 & -49.6 & -0.60 & {$[-212.81,113.59]$} & 0.06 \\
\hline \multirow{2}{*}{ Time spent } & Non-limit reaching gamblers & 376.5 & 273.2 & 310 & 135 & 389.0 & 382.0 & 277 & 153 & -32.6 & -2.10 & {$[-63.03,-2.13]$} & 0.08 \\
\hline & Limit reaching gamblers & 401.9 & 228.9 & 347 & 113 & 415.3 & 310.3 & 325 & 129 & 22.2 & -0.59 & {$[-96.01,51.59]$} & 0.06 \\
\hline \multirow{2}{*}{ Money lost } & Non-limit reaching gamblers & -52.5 & 143.9 & -38 & 43 & -68.3 & 212.1 & 58 & 52 & -20.1 & -3.50 & {$[-31.33,-8.85]$} & 0.14 \\
\hline & Limit reaching gamblers & -122.0 & 124.5 & -100 & 71 & -64.5 & 279.1 & -66 & 54 & 33.6 & 1.74 & {$[-4.17,71.37]$} & 0.19 \\
\hline \multirow{2}{*}{ Bets size } & Non-limit reaching gamblers & 7.7 & 4.4 & 7 & 3 & 7.7 & 5.1 & 6 & 3 & -0.32 & -1.01 & {$[-.93, .30]$} & 0.04 \\
\hline & Limit reaching gamblers & 8.8 & 4.5 & 9 & 3 & 8.5 & 5.1 & 7 & 3 & -1.5 & 0.93 & {$[-3.27, .36]$} & 0.10 \\
\hline
\end{tabular}

Note. MAD = Median absolute deviation. Loss limit in Multix ( $(-2500 \mathrm{NOK})$ Non-limit reaching gamblers $(\mathrm{n}=638)$, limit reaching gamblers $(\mathrm{n}=88)$. Money spent and net-balance in NOK. Time spent in

seconds. Average bet size $=$ Money spent $/$ bets made. $\Delta=$ Difference between $\mathrm{MD}$ in Non-alcohol serving venues and $\mathrm{MD}$ in Alcohol serving venues and subsequent significance test and $95 \% \mathrm{CI}$ of this difference. 